\title{
Peripheral Giant Cell Granuloma and Peripheral Ossifying Fibroma in Children: Two Case Reports
}

\author{
Nurhan Özalp ${ }^{a}$ Efsun Şener ${ }^{a}$ Timur Songur $^{b}$ \\ Departments of a Pedodontics and ${ }^{b}$ Oral and Maxillofacial Surgery, Faculty of Dentistry, Ankara University, \\ Ankara, Turkey
}

\section{Key Words}

Gingival lesions • Peripheral giant cell granuloma •

Peripheral ossifying fibroma

\begin{abstract}
Objectives: The aim of this study was to present the clinical and histopathological evaluation of reactive gingival lesions, such as peripheral giant cell granuloma (PGCG) and peripheral ossifying fibroma (POF), as exemplified in two cases. Clinical Presentation and Intervention: A 12- and a 13-year-old girl were referred to the Department of Pedodontics, both complaining of a painless swelling mass. After histopathological evaluation, the lesions were diagnosed as PGCG and POF. These gingival enlargements were totally resected after adequate clinical and radiological examinations. No recurrence has occurred in the 1-year follow-up period. Conclusion: These cases show that without histopathological evaluation, these two lesions could have been misdiagnosed due to their similarity in appearance. Early detection and treatment of these lesions are important to reduce bone loss or displacement of dental germs or teeth.
\end{abstract}

Copyright $\odot 2010$ S. Karger AG, Basel

\section{Introduction}

Reactive gingival lesions - such as peripheral giant cell granuloma (PGCG), peripheral ossifying fibroma (POF), parulis, hemangioma and pyogenic granuloma -resem- ble tumorous lesions, and they are usually localized to the gingiva (especially the interdental gingiva) or the alveolar mucosa. Reactive gingival lesions are often found to be associated with irritants such as plaque, calculus, poor dental restorations, ill-fitting dentures, trauma or tooth extraction; however, the etiology of these lesions is still not precisely known [1-2].

PGCG is an oral non-neoplastic tumorous proliferation that is usually localized to the gingiva or the alveolar mucosa without osseous tissue destruction [3]. Etiological factors of PGCG are tooth extraction, poor dental restorations, food impaction, ill-fitting dentures, plaque and calculus. Affected patients are generally adults and females in the fourth to sixth decades of life [3-4].

The radiographic findings of PGCG are generally unremarkable, although tooth or osseous resorption may rarely be found in an adjacent area [4]. Histologically, PGCGs present with giant cells in a vascular stroma of collagen fibers and are covered with stratified squamous epithelium. There are a large number of multinucleated giant cells resembling foreign body giant cells [5-6].

POF is a central bony lesion, although peripheral variations also exist $[1,7]$. It is a localized and relatively common gingival enlargement, occurring only on the gingiva and often related to the periodontal ligament. The anterior maxillary region is the most common localization for POFs (60\%). It is believed that inflammatory hyperplasia originating in the superficial periodontal ligament is a factor in the histogenesis of POF [2,7]. Although the exact

\section{KARGER \\ Fax +4161306 1234 E-Mail karger@karger.ch} www.karger.com

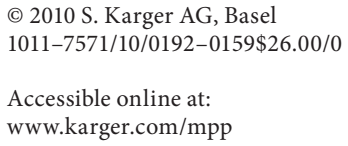

Nurhan Özalp

Department of Pedodontics, Faculty of Dentistry

Ankara University

TR-06500 Ankara (Turkey)

Tel. +90 312296 5668, Fax +90 312212 3954, E-Mail nurhan66@yahoo.com 


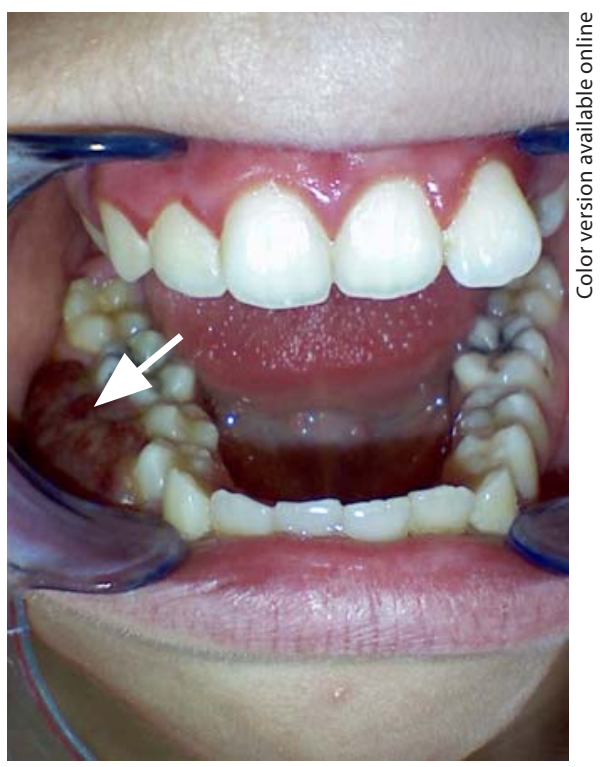

Fig. 1. Lingual displacement of second premolar and first molar teeth.

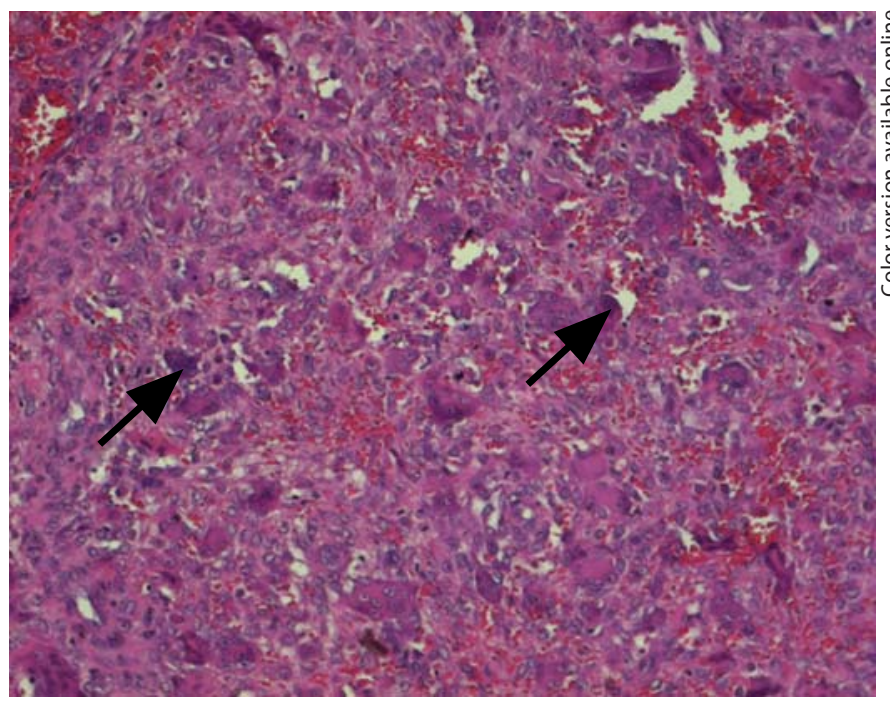

Fig. 2. Histological appearance of multinucleated giant cells in PGCG.

etiology is unknown, POFs are believed to be caused by irritants such as calculus, plaque, dental appliances, illfitting crowns and rough restorations. POFs usually occur between the ages of 25 and 35 years and 2-4 times more in females than males [7]. Interestingly, despite the reported occurrence of POF in children with primary or mixed dentition, there is very little documentation addressing the lesion's specific occurrence with primary teeth [8].

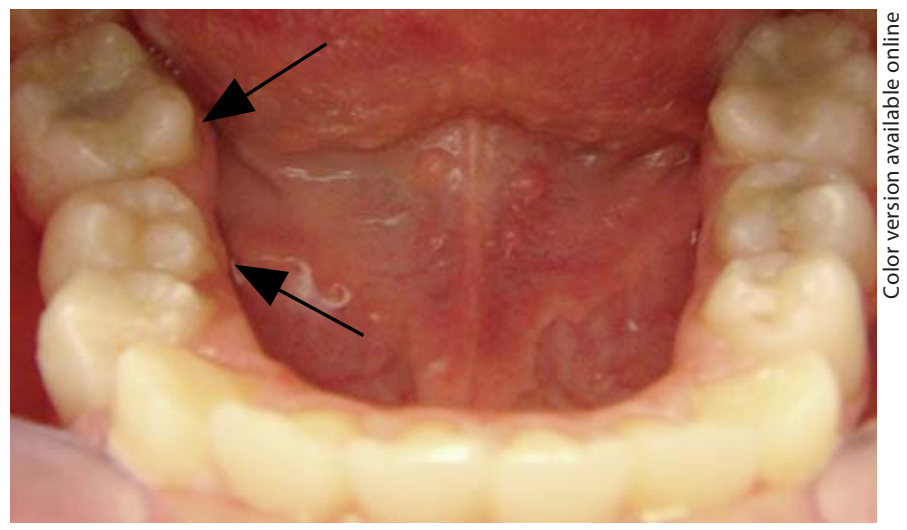

Fig. 3. One-year follow-up of case 1 .

PGCG and POF as gingival lesions occur commonly in adults although a few cases have been reported in children. We herein report a case of each that occurred in a 12- and a 13-year-old girl.

\section{Case 1}

A 12-year-old girl was referred to the Department of Pedodontics complaining of a swelling localized in the mandibular right premolar-molar region. According to the father, this lesion had appeared about a month before. The patient did not report pain, but complained about discomfort while chewing. No relevant medical data were found during the anamnesis. Intraoral clinical examination showed a reddish-purple tissue growth at the lower right gingival ridge in the premolar-molar region (fig. 1). The tissue bled upon palpation. The lesion was asymptomatic and measured approximately $3.5 \mathrm{~cm}$ in diameter. There was a lingual displacement of the molar region teeth that were affected by the gingival enlargement. The patient presented with deficient oral hygiene; hence, oral hygiene instructions were given. A panoramic radiograph was obtained that had no relevant findings, but a periapical radiograph at this site demonstrated superficial erosion of the alveolar bone. A biopsy and histopathological examination were performed.

Surgery was performed under local anesthesia; the mass was excised and the affected alveolar bone adjacent to the lesion was resected. The patient was given instructions about postoperative care, and a new appointment was made to remove the sutures. The diagnosis was PGCG, according to both the clinical and histopathological patterns (fig. 2). The adjacent mandibular teeth exhibited class II mobility with lingual displacement due to the presence of the gingival mass and the resected adjacent bone. The teeth were splinted for 2 months to prevent mobility, and the patient was examined 2 weeks following the procedure. The last follow-up, 1 year later, showed healthy attached gingiva in the molar region, no signs of recurrence and no mobility of adjacent teeth (fig. 3). Periapical radiographs at the site demonstrated new osseous formations. 


\section{Case 2}

A 13-year-old girl was referred to the Department of Pedodontics complaining of a swelling localized on the gingival mass in the anterior maxillary region. According to the patient, the 'reddish-purple lump' had been present for approximately 2 months, and her father stated that it had just recently become visible on the front teeth. As reported by the patient, the lump was interfering with her speech and felt uncomfortable. Occasionally, bleeding occurred when she brushed her teeth. Intraoral examination revealed a firm, sessile and erythematous papular swelling, located on the buccal gingiva of the left central incisor (fig. 4). The lesion was asymptomatic, nonulcerated and measured approximately $1.5 \mathrm{~cm}$ in diameter. There was no tooth displacement, and no root resorption related to the gingival enlargement was seen. A panoramic radiograph was obtained and determined within normal limits, with no findings pertaining to the maxillary exophytic lesion.

Surgery was performed under local anesthesia; the mass was completely excised and adjacent teeth were scaled to remove local irritants. The diagnosis was POF according to both clinical and histopathological patterns (fig. 5). The patient was followed up for 15 days postoperatively. The surgical site appeared to be healing well. There was no evidence of recurrence of the lesion over a 1year follow-up period.

\section{Discussion}

In children, gingival lesions that can imitate PGCG and POF are pyogenic granuloma, parulis, fibroma and peripheral odontogenic fibroma. On clinical examination, it is difficult to differentiate PGCG from POF $[1,2]$. Although POF is often ulcerated and inflamed, it lacks the purple or blue discoloration that is commonly associated with PGCG $[1,2]$. Histologically, POFs can be present with either an undamaged or ulcerated stratified squamous epithelium. The deeper fibroblastic component is highly cellular with central areas of calcification. The mineralized tissue may be composed of bone, cementum-like material, dystrophic calcification or a combination of each. POFs are more cellular than fibromas and less vascular than pyogenic granulomas $[1,2,8]$. Radiographically, POFs may exhibit diffused radiopaque calcifications, but not all lesions exhibit these features $[1$, $2,8]$.

Clinically, PGCG is a small (less than $1.5 \mathrm{~cm}$ ) welldemarcated reddish-blue focal mass on the gingiva, with a sessile or pedunculated base usually originating from either the periodontal ligament or mucoperiosteum [4-5, 8]. PGCG is mostly related to primary hyperparathyroidism [9]. A case of recurrent PGCG has been described by Stratakis et al. [10] in a 9-year-old boy affected by Xlinked hypophosphatemic rickets, a condition associated

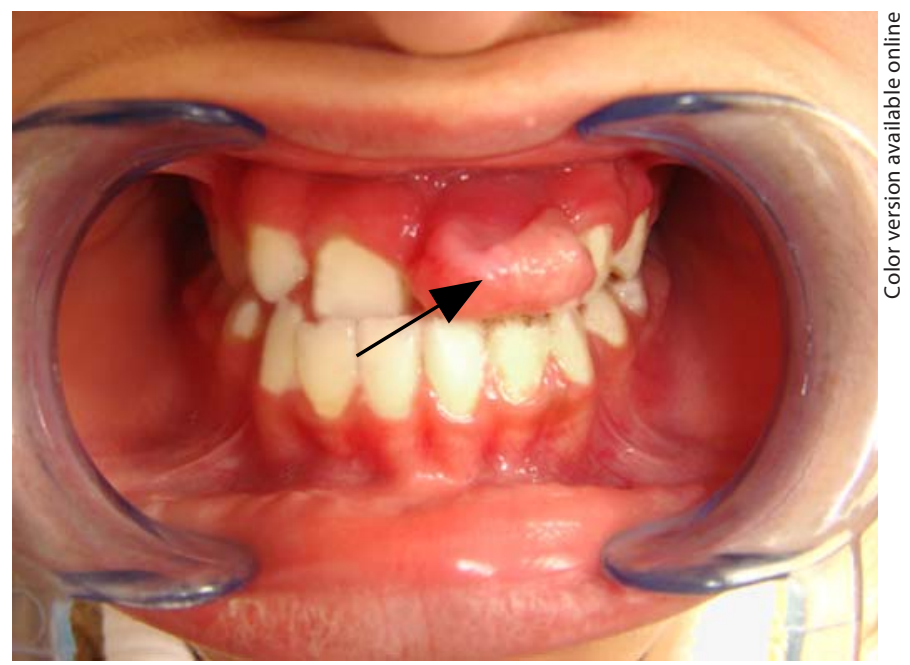

Fig. 4. Pedunculated nodule localized on the gingiva of left central and lateral incisor.

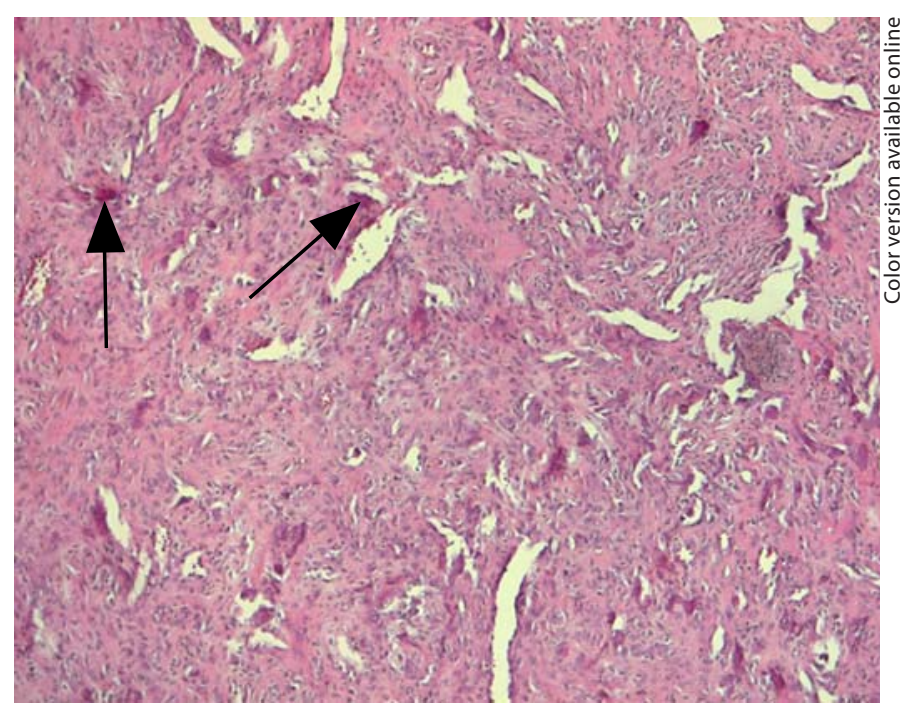

Fig. 5. Histological cross-section of POF.

with subclinical hyperparathyroidism. When it is important to exclude hyperparathyroidism, laboratory studies including serum calcium, phosphate, alkaline phosphatase and parathyroid hormone are required $[9,10]$. Recently, PGCG has been reported in a patient with glycogen storage disease type Ib [11].

Some authors have reported that PGCGs are more often located in the posterior region of the maxilla, but Katsikeris and Kakarantza-Angelopoulou [5] reported 
that the most common locations for PGCGs are the incisor and canine regions, with a predilection for the mandible. Contrary to other published reports, the PGCG in our first case occurred in the posterior region of the mandible.

PGCG is usually found in adults, and of the very few cases described in children, most were females [2]. Kfir et al. [1] and Zhang et al. [2] reported no difference in the incidence of PGCG between males and females. Although the radiographic findings for PGCGs are generally unremarkable, the displacement of teeth or bone defects may occur. Moreover, superficial resorption or cupping of the alveolar bone is usually observed on a periapical radiograph [3]. In case 1, the mandibular teeth adjacent to the PGCG exhibited class II mobility, with lingual displacement due to the presence of the gingival mass and resected adjacent bone. Flaitz [4] reported that a widened periodontal ligament space can be observed on a radiograph because of tooth mobility, but this can also be an extension of the lesion around the tooth. A periapical radiograph taken from case 1 demonstrated superficial erosion of the alveolar bone. Radiographic investigation should be done in all cases and is important for diagnosis.

The occurrence of this lesion associated with an orthodontic appliance was detected in $7 \%$ of pediatric cases described by Cuisia and Brannon [7]. Hanemann et al. [8] suggested that orthodontic appliances probably contributed to the etiopathogeny of the POF causing dental plaque or calculus accumulation. Although most lesions are less than $2 \mathrm{~cm}$ in size, Hanemann et al. [8] reported that the size of the lesion was usually smaller than $1.5 \mathrm{~cm}$ in diameter, as shown in case 2 .

In children, POF and PGCG can exhibit a rapid growth rate and reach a significant size in a relatively short period of time. In addition, these soft tissue nodules can cause superficial erosion of bone, produce minor or moderate tooth movement, and interfere with or delay eruption of teeth $[1,2,12]$. A biopsy, along with histopathological evaluation, is recommended for the definitive diagnosis of these lesions, and a proper treatment protocol is warranted with close follow-up [12]. Radiographs are important for diagnosis to confirm that these lesions arise in the oral mucosa and do not represent a central bony lesion with cortical perforation and soft tissue extension. Regardless of the surgical technique employed, it is important to eliminate etiological factors, including plaque, calculus and plaque-retentive restorations. Total excision of the involved periodontal ligament and periosteum is required to minimize the possibility of POF recurrence. Long-term follow-up is extremely important following surgical excision $[1,2$, 12]. Early detection of the POF and the PGCG results in more conservative surgery with less risk for tooth and osseous tissue loss.

\section{Conclusion}

Early detection and treatment of gingival lesions is important to reduce bone loss or displacement of dental germs or teeth.

\section{References}

1 Kfir Y, Buchner A, Hansen LS: Reactive lesions of the gingiva: a clinicopathological study of 741 cases. J Periodontol 1980;51: 655-661.

-2 Zhang W, Chen Y, An Z, Geng N, Bao D: Reactive gingival lesions: a retrospective study of 2,439 cases. Quintessence Int 2007;38: $103-110$.

- 3 Bodner L, Peist M, Gatot A, Fliss DM: Growth potential of peripheral giant cell granuloma. Oral Surg Oral Med Oral Pathol Oral Radiol Endod 1997;83:548-551.

-4 Flaitz CM: Peripheral giant cell granuloma: a potentially aggressive lesion in children. Pediatr Dent 2000;22:232-233.
5 Katsikeris N, Kakarantza-Angelopoulou E: Peripheral giant cell granuloma: clinicopathologic study of 224 new cases and 956 reported cases. Int J Oral Maxillofac Surg 1988;17:94-99.

6 Eronat N, Aktug M, Giinbay T, Unal T: Peripheral giant cell granuloma: three case reports. J Clin Pediatr Dent 2000;24:245-248.

7 Cuisia ZE, Brannon RB: Peripheral ossifying fibroma: a clinical evaluation of 134 pediatric cases. Pediatr Dent 2001;23:245-248.

8 Hanemann JA, Pereira AA, Ribeiro Júnior NV, Oliveira DT: Peripheral ossifying fibroma in a child: report of case. J Clin Pediatr Dent 2003;27:283-285.

-9 Attie JN, Blum B: Hyperparathyroidism first suspected through a giant cell epulis: report of a case. Oral Surg Oral Med Oral Pathol 1960;13:482-493.
10 Stratakis CA, Mitsiades NS, Chrousos GP, O'Connell A: Recurring oral giant cell lesion in a child with X-linked hypophosphatemic rickets: clinical manifestation of occult parathyroidism. J Pediatr 1995;127:444-446.

11 Mortellaro C, Garagiola U, Carbone V, Cerutti F, Marci V, Bonda PL: Unusual oral manifestations and evolution in glycogen storage disease type Ib. J Craniofac Surg 2005; 16:45-52.

12 Pinkham JR: Oral pathologic conditions and soft tissue anomalies in children; in Pinkham JR, Casamassimo PS, Fields HW Jr, McTigue DJ, Nowak A (eds): Pediatric Dentistry, Infancy through Adolescence. Philadelphia, WB Saunders, 1988, vol 40, pp 9-61. 